\title{
Comparative In Vitro Activities of Gemifloxacin, Other Quinolones, and Nonquinolone Antimicrobials against Obligately Anaerobic Bacteria
}

\author{
NIELS KLEINKAUF, ${ }^{*}$ GRIT ACKERMANN, REINER SCHAUMANN, AND ARNE C. RODLOFF \\ Institute for Medical Microbiology and Epidemiology of Infectious Diseases, University of Leipzig, Leipzig, Germany
}

Received 27 November 2000/Returned for modification 17 January 2001/Accepted 6 March 2001

\begin{abstract}
The in vitro activity of gemifloxacin was compared to that of other quinolone and nonquinolone antimicrobials against 204 anaerobes by the agar dilution technique. The data indicate that gemifloxacin has a rather selective anaerobic activity. Most Peptostreptococcus, Porphyromonas, and Fusobacterium species are susceptible, while gemifloxacin's activity against other gram-negative anaerobes appears to be variable.
\end{abstract}

Gemifloxacin mesylate (SB 265805) is a fluoroquinolone with a novel oxime-derivatized pyrrolidine substituent at position $\mathrm{C} 7$ which is thought to confer the enhanced activity against gram-positive bacteria (11). A limited number of studies have focused on its potential activity against anaerobic bacteria, demonstrating variable susceptibility patterns of the different anaerobic genera $(3,4,7-10,12)$. To further evaluate its utility in the treatment of infections involving anaerobes, we studied the in vitro activity of gemifloxacin and several comparator antimicrobials against selected anaerobic isolates from clinical specimens obtained in Germany.

The 204 anaerobic strains used in this study were recent clinical isolates (collected from September 1997 through March 2000) from our institute, the Department of Medical Microbiology of the University of Tübingen, Tübingen, Germany, and the Institute of Medical Microbiology of the University of Halle, Halle, Germany. The numbers and species of isolates tested are given in Table 1 . The bacterial strains were identified by use of prereduced anaerobically sterilized (PRAS) biochemicals (Carr Scarborough Microbiologicals, Stone Mountain, Ga.) to test for fermentation of arabinose, rhamnose, trehalose, salicin, sucrose, and xylan; hydrolysis of esculin; and production of indole. Bile resistance was determined by growth in PRAS peptone-yeast broth containing $20 \%$ bile. Identification schemes were followed as detailed previously by Claros et al. (1, 2). In addition, RapID ANA II (Innovative Diagnostic Systems, Norcross, Ga.) and, in some cases, ATB 32A (bioMérieux, Marcy l'Etoile, France) were inoculated, and the results were interpreted according to the manufacturer's instructions.

Susceptibilities were determined by an agar dilution method according to the National Committee for Clinical Laboratory Standards (NCCLS) using brucella agar (Difco Laboratories, Detroit, Mich.) supplemented with hemin of $5 \mu \mathrm{g} / \mathrm{ml}$, vitamin $\mathrm{K}$ at $1 \mu \mathrm{g} / \mathrm{ml}$, and $5 \%$ laked horse blood (Oxoid $\mathrm{GmbH}$, Wesel, Germany) (13). Serial doubling dilutions (0.03 to $32 \mathrm{mg} / \mathrm{liter}$ )

\footnotetext{
* Corresponding author. Mailing address: University of Leipzig, Institute for Medical Microbiology and Epidemiology of Infectious Diseases, Liebigstr. 24, D-04103 Leipzig, Germany. Phone: 49 (341) 9715200. Fax: 49 (341) 9715209. E-mail: klen@medizin.uni-leipzig.de.
}

were prepared for all compounds tested. Dilutions of the antibiotics were made according to standard procedures on the day of the test. Inocula were prepared according to NCCLS standards (13). The final inoculum density was calculated to be approximately $10^{5} \mathrm{CFU} /$ spot. The individual inocula were applied by use of a semiautomatic replicator device (A400 Multipoint Inoculator; Bachhofer GmbH, Reutlingen, Germany). The MICs were determined after incubation in an anaerobic chamber (WA 6600; Heraeus Instruments, Hanau, Germany) containing an atmosphere of $80 \% \mathrm{~N}_{2}, 15 \% \mathrm{CO}_{2}$, and $5 \% \mathrm{H}_{2}$ for $48 \mathrm{~h}$ at $37^{\circ} \mathrm{C}$. Bacteroides fragilis ATCC 25285 and Bacteroides thetaiotaomicron ATCC 29741 served as controls to monitor the stability of the antimicrobial preparations. Antimicrobial reference powders were as follows: gemifloxacin and amoxicillin-clavulanate (2:1) (SmithKline Beecham Pharma $\mathrm{GmbH}$, Munich, Germany); moxifloxacin (Bayer, Leverkusen, Germany); penicillin G (Serva, Heidelberg, Germany); imipenem (MSD, Haar, Germany); and clindamycin and metronidazole (Sigma, Steinheim, Germany).

The $\mathrm{MIC}_{90} \mathrm{~s}$ of gemifloxacin were equal to or one to two dilutions lower than those of moxifloxacin against various species of Peptostreptococcus. The $\mathrm{MIC}_{90} \mathrm{~s}$ for Peptostreptococcus asacharolyticus, $P$. micros, and $P$. prevotii were between 0.125 and $0.25 \mathrm{mg} /$ liter. However, some isolates of $P$. anaerobius and $P$. magnus were resistant, resulting in gemifloxacin $\mathrm{MIC}_{90} \mathrm{~s}$ of 0.5 and $4 \mathrm{mg} / \mathrm{liter}$, respectively, indicating that the activity of gemifloxacin is not entirely predictable, even against grampositive anaerobes, and that there is species variability in its activity against peptostreptoccoci. Goldstein et al. (8) noted far lower $\mathrm{MIC}_{90}$ s for these species $(0.03$ and $0.06 \mathrm{mg} /$ liter, respectively). Interestingly, the MIC ranges at least of $P$. anaerobius indicate that Goldstein similarly found isolates for which the MICs were up to $8 \mathrm{mg} /$ liter, so that the differences may be explained by the significantly smaller number of isolates tested in Goldstein's study (13 versus 23 and 14 versus 24 isolates of $P$. anaerobius and $P$. magnus, respectively). Differences in local susceptibility patterns may further contribute to the discrepancies.

Gemifloxacin was active against $B$. fragilis at $\leq 4 \mathrm{mg} /$ liter with an MIC range of 0.5 to $4 \mathrm{mg} /$ liter. Similar results were reported by Goldstein et al. (8), who found an $\mathrm{MIC}_{90}$ of $2 \mathrm{mg} /$ liter and 
TABLE 1. In vitro activity of gemifloxacin and other quinolone and non-quinolone antimicrobials against obligately anaerobic bacteria

\begin{tabular}{|c|c|c|c|c|c|c|c|c|c|c|c|c|c|c|}
\hline \multirow{2}{*}{$\begin{array}{l}\text { Organism (no. of isolates) and } \\
\text { antimicrobial agent }\end{array}$} & \multicolumn{12}{|c|}{ No. of isolates for which the MIC (mg/liter) was: } & \multicolumn{2}{|c|}{ MIC (mg/liter) } \\
\hline & $\leq 0.03$ & 0.06 & 0.125 & 0.25 & 0.5 & 1 & 2 & 4 & 8 & 16 & 32 & $>32$ & $\mathrm{MIC}_{50}$ & $\mathrm{MIC}_{90}$ \\
\hline \multicolumn{15}{|l|}{ Bacteroides fragilis (26) } \\
\hline Gemifloxacin & & & & & 5 & 17 & 1 & 3 & & & & & 1 & 4 \\
\hline Moxifloxacin & & & & 5 & 15 & 4 & 2 & & & & & & 0.5 & 1 \\
\hline Penicillin G & & & & & & & & & & 5 & 20 & 1 & 32 & 32 \\
\hline Amoxicillin-clavulanate & & & 8 & 15 & 1 & 2 & & & & & & & 0.25 & 0.5 \\
\hline Imipenem & 6 & 17 & 3 & & & & & & & & & & 0.06 & 0.125 \\
\hline Metronidazole & & & & & 9 & 14 & 3 & & & & & & 1 & 2 \\
\hline Clindamycin & & 1 & 1 & 1 & 12 & 5 & & & & 1 & 5 & & 0.5 & 32 \\
\hline \multicolumn{15}{|l|}{ Fusobacterium spp. (24) } \\
\hline Gemifloxacin & 1 & 1 & 4 & 8 & 8 & 2 & & & & & & & 0.25 & 0.5 \\
\hline Moxifloxacin & & & 4 & 6 & 3 & 5 & 4 & & 2 & & & & 0.5 & 2 \\
\hline Penicillin G & 11 & 3 & 3 & 2 & 2 & & & 1 & & & 1 & 1 & 0.06 & 4 \\
\hline Amoxicillin-clavulanate & 20 & & & 2 & 1 & & 1 & & & & & & $\leq 0.03$ & 0.25 \\
\hline Imipenem & 7 & 10 & 3 & 1 & 3 & & & & & & & & 0.06 & 0.5 \\
\hline Metronidazole & 1 & 3 & 7 & 5 & 4 & & 2 & & & & & 2 & 0.25 & 2 \\
\hline Clindamycin & 14 & 4 & 2 & 1 & & 2 & & 1 & & & & & $\leq 0.03$ & 1 \\
\hline \multicolumn{15}{|l|}{ Peptostreptococcus anaerobius (23) } \\
\hline Gemifloxacin & 1 & & 17 & 2 & 1 & & 1 & & 1 & & & & 0.125 & 0.5 \\
\hline Moxifloxacin & & 1 & 15 & 3 & 1 & 1 & 1 & 1 & & & & & 0.125 & 1 \\
\hline Penicillin G & & 1 & 1 & 16 & 4 & & & 1 & & & & & 0.25 & 0.5 \\
\hline Amoxicillin-clavulanate & 2 & 1 & 3 & 11 & 5 & 1 & & & & & & & 0.25 & 0.5 \\
\hline Imipenem & 23 & & & & & & & & & & & & $\leq 0.03$ & $\leq 0.03$ \\
\hline Metronidazole & & & 1 & 5 & 12 & 1 & 3 & & & & 1 & & 0.5 & 2 \\
\hline Clindamycin & 6 & 7 & 7 & 1 & 2 & & & & & & & & 0.06 & 0.25 \\
\hline \multicolumn{15}{|c|}{ Peptostreptococcus asaccharolyticus (22) } \\
\hline Gemifloxacin & 3 & 2 & 11 & 4 & 2 & & & & & & & & 0.125 & 0.25 \\
\hline Moxifloxacin & & & 2 & 6 & 9 & 1 & 3 & 1 & & & & & 0.5 & 2 \\
\hline Penicillin G & 11 & & 4 & 3 & 2 & 2 & & & & & & & 0.03 & 1 \\
\hline Amoxicillin-clavulanate & 13 & 1 & 3 & 4 & 1 & & & & & & & & 0.03 & 0.25 \\
\hline Imipenem & 13 & 4 & 2 & 2 & 1 & & & & & & & & 0.03 & 0.25 \\
\hline Metronidazole & & 1 & 2 & 4 & 6 & 9 & & & & & & & 1 & 2 \\
\hline Clindamycin & 2 & 6 & 9 & 2 & & 1 & & & & & & 2 & 0.12 & 1 \\
\hline \multicolumn{15}{|l|}{ Peptostreptococcus magnus (24) } \\
\hline Gemifloxacin & 5 & 12 & 1 & & & 3 & & 1 & 1 & 1 & & & 0.06 & 4 \\
\hline Moxifloxacin & & 2 & 8 & 7 & 2 & 1 & & 1 & 1 & 1 & 1 & & 0.25 & 4 \\
\hline Penicillin G & & 2 & 4 & 13 & 4 & & & 1 & & & & & 0.25 & 0.5 \\
\hline Amoxicillin-clavulanate & & 2 & 5 & 12 & 5 & & & & & & & & 0.25 & 5 \\
\hline Imipenem & & 7 & 8 & 8 & 1 & & & & & & & & 0.125 & 0.25 \\
\hline Metronidazole & & & 1 & 5 & 8 & 8 & 2 & & & & & & 0.5 & 1 \\
\hline Clindamycin & & 3 & 7 & 5 & 5 & 1 & 1 & & & & & 2 & 0.25 & 2 \\
\hline \multicolumn{15}{|l|}{ Peptostreptococcus micros (24) } \\
\hline Gemifloxacin & 6 & 1 & 17 & & & & & & & & & & 0.125 & 0.125 \\
\hline Moxifloxacin & 1 & & 1 & 22 & & & & & & & & & 0.25 & 0.25 \\
\hline Penicillin G & 21 & 2 & & 1 & & & & & & & & & $\leq 0.03$ & 0.06 \\
\hline Amoxicillin-clavulanate & 21 & 1 & & 2 & & & & & & & & & $\leq 0.03$ & 0.06 \\
\hline Imipenem & 3 & 19 & 2 & & & & & & & & & & 0.06 & 0.06 \\
\hline Metronidazole & 7 & 5 & 3 & 7 & 2 & & & & & & & & 0.125 & 0.25 \\
\hline Clindamycin & 1 & & 3 & 13 & 6 & & & 1 & & & & & 0.25 & 0.5 \\
\hline \multicolumn{15}{|l|}{ Peptostreptococcus prevotii (20) } \\
\hline Gemifloxacin & 7 & 7 & 4 & 2 & & & & & & & & & 0.06 & 0.125 \\
\hline Moxifloxacin & & & 1 & 9 & & 1 & 7 & 1 & & 1 & & & 0.25 & 2 \\
\hline Penicillin G & & 5 & 11 & 3 & 1 & & & & & & & & 0.125 & 0.25 \\
\hline Amoxicillin-clavulanate & 13 & & 1 & 6 & & & & & & & & & $\leq 0.03$ & 0.25 \\
\hline Imipenem & 13 & 3 & 4 & & & & & & & & & & $\leq 0.03$ & 0.125 \\
\hline Metronidazole & & & 7 & 1 & 3 & 8 & 1 & & & & & & 0.5 & 1 \\
\hline Clindamycin & 4 & 6 & 2 & 3 & & 3 & 1 & & & & 1 & & 0.06 & 1 \\
\hline \multicolumn{15}{|l|}{ Porphyromonas $^{b}$ spp. (20) } \\
\hline Gemifloxacin & 9 & 4 & 7 & & & & & & & & & & 0.06 & 0.125 \\
\hline Moxifloxacin & 18 & 2 & & & & & & & & & & & $\leq 0.03$ & $\leq 0.03$ \\
\hline Penicillin G & 20 & & & & & & & & & & & & $\leq 0.03$ & $\leq 0.03$ \\
\hline
\end{tabular}


TABLE 1-Continued

\begin{tabular}{|c|c|c|c|c|c|c|c|c|c|c|c|c|c|c|}
\hline \multirow{2}{*}{$\begin{array}{l}\text { Organism (no. of isolates) and } \\
\text { antimicrobial agent }\end{array}$} & \multicolumn{12}{|c|}{ No. of isolates for which the MIC (mg/liter) was: } & \multicolumn{2}{|c|}{ MIC (mg/liter) } \\
\hline & $\leq 0.03$ & 0.06 & 0.125 & 0.25 & 0.5 & 1 & 2 & 4 & 8 & 16 & 32 & $>32$ & $\mathrm{MIC}_{50}$ & $\mathrm{MIC}_{90}$ \\
\hline Amoxicillin-clavulanate & 20 & & & & & & & & & & & & $\leq 0.03$ & $\leq 0.03$ \\
\hline Imipenem & 20 & & & & & & & & & & & & $\leq 0.03$ & $\leq 0.03$ \\
\hline Metronidazole & 20 & & & & & & & & & & & & $\leq 0.03$ & $\leq 0.03$ \\
\hline Clindamycin & 20 & & & & & & & & & & & & $\leq 0.03$ & $\leq 0.03$ \\
\hline \multicolumn{15}{|l|}{ Prevotella $^{c}$ spp (21) } \\
\hline Gemifloxacin & 1 & & & 2 & 2 & 13 & 2 & & 1 & & & & 1 & 2 \\
\hline Moxifloxacin & 1 & & & 2 & 12 & 4 & 2 & & & & & & 0.5 & 1 \\
\hline Penicillin G & 1 & 2 & 10 & 1 & & & 1 & 3 & 1 & & & 2 & 0.125 & 8 \\
\hline Amoxicillin-clavulanate & 9 & 8 & 2 & 2 & & & & & & & & & 0.06 & 0.125 \\
\hline Imipenem & 18 & 3 & & & & & & & & & & & $\leq 0.03$ & 0.06 \\
\hline Metronidazole & & 1 & & 3 & 8 & 3 & 2 & 3 & & & 1 & & 0.5 & 4 \\
\hline Clindamycin & 20 & & & & & & & & & & & 1 & 0.015 & 0.03 \\
\hline
\end{tabular}

${ }^{a}$ That is, Fusobacterium necrophorum (16), F. nucleatum (6), F. mortiferum (1), and F. varium (1).

${ }^{b}$ That is, Porphyromonas gingivalis (13), P. endodontalis (3), P. asaccharolytica (2), and P. levii (2).

${ }^{c}$ That is, Prevotella melaninogenica (10), P. oris (4), P. intermedia (3), P. bivia (1), P. buccae (1), P. denticola (1), and P. disiens (1).

a range of 0.5 to $2 \mathrm{mg} /$ liter. Marco et al. (12), who tested 35 isolates of $B$. fragilis, reported an MIC range of 0.5 to $8 \mathrm{mg} /$ liter and an $\mathrm{MIC}_{90}$ of $1 \mathrm{mg} /$ liter. Wise and Andrews (14) originally proposed a gemifloxacin breakpoint of $0.5 \mathrm{mg} /$ liter based on the formula of the British Society for Antimicrobial Chemotherapy. Recently, even more conservative criteria using a $\leq 0.25$-mg/liter susceptible MIC breakpoint have been proposed (5). Yet, already with the previously proposed criteria, a large percentage of $B$. fragilis strains would be resistant.

As indicated by the wide range of MIC values, Prevotella spp. exhibited variable susceptibilities against gemifloxacin. Goldstein et al. (8) tested a large number of Prevotella isolates and reported marked differences between various Prevotella spp. Most of the isolates tested in our study were Prevotella melaninogenica and $P$. intermedia, corresponding to the more resistant species of the isolates tested by Goldstein et al. Therefore, discrepancies between the studies in the overall activity of gemifloxacin against Prevotella may be explained by the marked differences in susceptibility of the various species.

All Porphyromonas isolates tested, including strains of Porphyromonas gingivalis (13), P. endodontalis (3), P. asaccharolytica (2), and $P$. levii (2), were inhibited by $\leq 0.125 \mathrm{mg}$ of gemifloxacin per liter.

Most of the fusobacteria in our study were also susceptible to gemifloxacin, with an $\mathrm{MIC}_{90}$ of $0.5 \mathrm{mg}$ /liter. The activity of gemifloxacin against fusobacteria was one to two dilutions lower than that of moxifloxacin. The majority of strains included in our study were Fusobacterium necrophorum (16 of 24 ) and F. nucleatum (6 of 24). Goldstein et al. (9) reported similar $\mathrm{MIC}_{90}$ values for these species $(0.5$ and $0.25 \mathrm{mg} /$ liter, respectively).

All anaerobes tested in this study were susceptible to the comparator antimicrobials imipenem and amoxicillin-clavulanate according to NCCLS criteria (13). For metronidazole and clindamycin, the overall susceptibility rates were 98 and $93 \%$, respectively. As judged by the difference between penicillin and amoxicillin-clavulanate susceptibility, $26 \%$ of the isolates were lactamase producers.

In conclusion, the antimicrobial potency of gemifloxacin against anaerobic bacteria appears to be variable. In accor- dance with previously published studies, the majority of the Peptostreptococcus and Porphyromonas strains appear to be susceptible. Yet the present study shows that unequal susceptibilities between species of the same genus exist. Furthermore, possible variations between local susceptibility patterns must be taken into account when the use of gemifloxacin in treating anaerobic infections by these species is considered. Compared to the general resistance of fusobacteria toward the older quinolones (6), gemifloxacin clearly shows enhanced activity against these pathogens. Against other gram-negative anaerobes gemifloxacin exhibits only moderate activity. Considered in light of the complex nature of most anaerobic infections, the data presented here must be regarded as preliminary, and further clinical studies appear warranted to evaluate the role of gemifloxacin in such infections.

\section{ACKNOWLEDGMENTS}

We thank U. Schumacher, University of Tübingen, Tübingen, Germany, and C. D. Höhne, University of Halle, Halle, Germany, for supplying bacterial strains for this study.

This study was funded in part by SmithKline Beecham, Munich, Germany.

\section{REFERENCES}

1. Claros, M. C., D. M. Citron, S. Hunt-Gerardo, E. J. Goldstein, G. Schönian, T. Montag, B. Hampel, and A. C. Rodloff. 1996. Characterization of indolenegative Bacteroides fragilis group species with use of polymerase chain reaction fingerprinting and resistance profiles. Clin. Infect. Dis. 23(Suppl. 1):S66-S72.

2. Claros, M. C., Y. Papke, N. Kleinkauf, D. Adler, D. M. Citron, S. HuntGerardo, T. Montag, E. J. C. Goldstein, and A. C. Rodloff. 1999. Characteristics of Fusobacterium ulcerans, a new and unusual species compared with Fusobacterium varium and Fusobacterium mortiferum. Anaerobe 5:137-140.

3. Cormican, M. G., and R. N. Jones. 1997. Antimicrobial activity and spectrum of LB20304, a novel fluoronaphthyridone. Antimicrob. Agents Chemother 41:204-211.

4. Dubois, J., and C. St-Pierre. 2000. Comparative in vitro activity and postantibiotic effect of gemifloxacin against Legionella spp. J. Antimicrob. Chemother. 45(Suppl.1):41-46.

5. Fuchs, P. C., A. L. Barry, and S. D. Brown. 2000. In vitro activity of gemifloxacin against contemporary clinical bacterial isolates from eleven North American medical centers, and assessment of disk diffusion test interpretive criteria. Diagn. Microbiol. Infect. Dis. 38:243-253.

6. Goldstein, E. J., D. M. Citron, M. Hudspeth, S. Hunt-Gerardo, and C. V. Merriam. 1997. In vitro activity of Bay 12-8039, a new 8-methoxyquinolone, 
compared to the activities of 11 other oral antimicrobial agents against 390 aerobic and anaerobic bacteria isolated from human and animal bite wound skin and soft tissue infections in humans. Antimicrob. Agents Chemother. 41:1552-1557.

7. Goldstein, E. J., D. M. Citron, C. V. Merriam, K. Tyrrell, and Y. Warren. 1999. Activities of gemifloxacin (SB 265805, LB20304) compared to those of other oral antimicrobial agents against unusual anaerobes. Antimicrob. Agents Chemother. 43:2726-2730.

8. Goldstein, E. J., D. M. Citron, Y. Warren, K. Tyrrell, and C. V. Merriam. 1999. In vitro activity of gemifloxacin (SB 265805) against anaerobes. Antimicrob. Agents Chemother. 43:2231-2235.

9. Goldstein, E. J. 2000. Review of the in vitro activity of gemifloxacin against gram-positive and gram-negative anaerobic pathogens. J. Antimicrob. Chemother. 45(Suppl. 1):55-65.
10. King, A., J. May, G. French, and I. Phillips. 2000. Comparative in vitro activity of gemifloxacin. J. Antimicrob. Chemother. 45(Suppl. 1):1-12.

11. MacGowan, A. P. 1999. SB-265805 (SB)-a potent new quinolone. Clin. Microbiol. Infect. 5(Suppl. 3):20.

12. Marco, F., M. S. Barrett, and R. N. Jones. 1997. Antimicrobial activity of LB20304, a fluoronaphthyridone, tested against anaerobic bacteria. J. Antimicrob. Chemother. 40:605-607.

13. National Committee for Clinical Laboratory Standards. 1997. Methods for antimicrobial susceptibility testing of anaerobic bacteria, 4th ed. National Committee for Clinical Laboratory Standards, Villanova, Pa.

14. Wise, R., and J. M. Andrews. 1999. The in vitro activity and tentative breakpoint of gemifloxacin, a new fluoroquinolone. J. Antimicrob. Chemother. 44:679-688. 